\title{
CONEXIONES ENTRE PENSIÓN 65 Y LA POBREZA EN LOS ADULTOS MAYORES: PERÚ 2012-2018
}

\author{
CONNECTIONS BETWEEN PENSION 65 AND ELDERLY POVERTY: PERU 2012-2018
}

Alipio Orco Díaz

Universidad Nacional Micaela Bastidas de Apurímac

Abancay, Perú

ORCID: https://orcid.org/0000-0003-1307-0422

Correo electrónico: aorco@unamba.edu.pe

Karla Sadith Santa Cruz Vargas Universidad Nacional Micaela Bastidas de Apurímac Abancay, Perú ORCID: https://orcid.org/0000-0001-8529-0219 Correo electrónico: ksanta@unamba.edu.pe

Miguel Ángel Juro Llamocca Universidad Nacional Micaela Bastidas de Apurímac Abancay, Perú

ORCID: https://orcid.org/0000-0002-9169-3505 Correo electrónico: mjuro@unamba.edu.pe

[Recibido: 10/08/2020 Aceptado: 10/12/2020 Publicado: 28/12/2020]

\section{RESUMEN}

Objetivo: Determinar la relación del Programa Pensión 65 con el nivel de pobreza en los adultos mayores en el Perú: periodo 2012-2018. Método: La investigación fue de tipo cuantitativo no experimental y longitudinal; de alcance descriptivo, correlacional y explicativo; la población objeto de estudio fueron las personas mayores en situación de pobreza, beneficiarias del Programa Pensión 65 y que fueron comprendidas en la Encuesta Nacional de Hogares (ENAHO) en el periodo 2012-2018. Resultados: Se demostró que los beneficiarios del Programa Pensión 65 tienen una probabilidad de $11 \%$ más de salir de la pobreza frente a los adultos mayores que no reciben Pensión 65; los hogares jefaturados por mujeres tienen $0,34 \%$ más de probabilidad de ser pobres en relación a los hogares jefaturados por hombres; vivir en el área urbana reduce la probabilidad de ser pobre en $19,4 \%$, respecto a los que viven en el área rural; un aumento en un año de edad, aumenta en $0,27 \%$ la probabilidad de ser pobre, manteniendo constantes las otras variables; un nivel educativo adicional reduce en $11 \%$ la probabilidad de ser pobre respecto al nivel anterior. Conclusiones: El programa de pensión no contributiva, Pensión 65, constituye una política pública eficaz y eficiente para disminuir el nivel de pobreza y vulnerabilidad en la vejez en el Perú.

\begin{abstract}
Objective: Determine the relationship of the Pension 65 Program with the level of elderly poverty in Peru: period 2012-2018. Method: The research was of a non-experimental and longitudinal quantitative type; descriptive, correlational and explanatory scope. The population studied was the elderly people in poverty situation, Pension 65 program beneficiaries and who were included in the National Household survey (ENAHO) in the period $2012-2018$. Results: It demonstrated that Pension 65 program beneficiaries had a $11 \%$ more of probability, to get out of poverty compare to older adults who do not receive Pension 65 ; the house head by women has $0,34 \%$ more of probability to be poor related to the houses head by men; live in the urban areas reduces the probability of being poor in $19.4 \%$, compare to those who live in the rural areas. an increase in one year of age increases the probability of being poor in $0,27 \%$, keeping the other variables constant; an additional level of education reduces the probability of being poor in $11 \%$ respect from the previous level. Conclusions: The non-contributory pension program, Pension 65, is an effective and efficient public policy to reduce the level of poverty and vulnerability in elderly in Peru.
\end{abstract}

Palabras clave: Adultos mayores; pensión 65; pensiones no contributivas; pobreza.

Keywords: Elderly; pension 65; non-contributory pensions; poverty.

( ) Los autores. Este artículo es publicado por la Revista Quipukamayoc, Universidad Nacional Mayor de San Marcos. Este es un artículo de acceso abierto, distribuido bajo los términos de la Licencia Creative Commons Atribución-NoComercial-Compartirlgual 4.0 Internacional.(http://creativecommons.org/licenses/by-nc-sa/4.0/), que permite el uso no comercial, distribución y reproducción en cualquier medio, siempre que la obra original sea debidamente citadas. 


\section{INTRODUCCIÓN}

En estos tiempos de emergencia sanitaria por el COVID-19, el debate público y político está en torno, entre otros temas, al sistema de pensiones, retiros del $25 \%$ de las Administradoras de Fondos de Pensiones (AFP), propuestas de retiros de la ONP y una creciente conciencia social de reformar el Sistema Nacional de Pensiones en el Perú. Llegar a la vejez en situación de pobreza es una real posibilidad, para el 63\% de adultos mayores del país; como consecuencia de una precaria trayectoria laboral, sobre todo para las personas que trabajan en la informalidad, en empleos escasamente remunerados o autoempleos poco rentables y sin cotizar al sistema de pensiones. El que ha sido pobre en su edad económicamente activa tendrá una alta probabilidad de llegar en situación de pobreza o pobreza extrema a la etapa de la vejez.

La pobreza es un término polisémico, por lo mismo entendida desde diversos enfoques, que en algunos casos se superponen unos sobre otros. Pero de manera general, estos enfoques se complementan, dado que tocan diversas aristas de un mismo problema. Para efectos de este trabajo, se emplea el enfoque monetario de la pobreza, que conceptualiza la pobreza como la expresión de la falta, carencia o insuficiencia de ingresos monetarios; situación que imposibilita la adquisición de una canasta básica de consumo, que comprende alimentos y servicios. Este enfoque permite identificar a los pobres entre el total de una población en función a un umbral de ingresos, equivalente al valor de una canasta básica de consumo y las personas cuyos ingresos se ubiquen por debajo de ese umbral se consideran como pobres (Castro, 2013).

El tema objeto de esta investigación tiene relación con la teoría del Ciclo de Vital desarrollado por el economista Franco Modigliani, Premio Nobel de Economía, 1985.

La hipótesis central de esta teoría sostiene que cuando la persona es joven, sus ingresos son menores al nivel de consumo deseado, por lo que generalmente se endeuda, dado que supone que sus ingresos se incrementarán conforme avance su edad. Posteriormente, en la edad adulta, sus ingresos son mayores y le permiten cubrir su consumo, pagar sus deudas de periodos anteriores y ahorrar para la vejez. Al jubilarse, sus ingresos caerán al nivel cero y deben financiar su consumo con sus ahorros anteriores (Modigliani, 1986). La función consumo que expresa la esencia de esta teoría se plasma en la siguiente ecuación:

$$
C_{\mathrm{t}}=\alpha_{1} y_{t}+\alpha_{2} y_{t}^{e}+\alpha_{3} A_{t-1}
$$

Donde $c$ es el consumo agregado; $y$ representa el ingreso corriente no proveniente de la propiedad; $y^{e}$ es el ingreso anual esperado no proveniente de la propiedad; $A$ representa la riqueza neta; $\alpha_{1}, \alpha_{2}, \alpha_{3}$ son la propensión marginal por consumir; $t$ representa la cantidad de años que se espera vivir. La idea principal que se desprende de este modelo es que el consumo está relacionado con los ingresos que recibirá durante toda su vida laboral y no con el ingreso corriente.

El enfoque económico del ciclo vital se complementa con el enfoque desde una perspectiva biológica y psicosocial que afirma que el hombre, por su propia naturaleza, experimenta un permanente transcurrir de la vida, por distintas etapas: niñez, adolescencia, juventud, adultez y vejez. En el marco de esta forma de comprender la existencia humana, Ocampo y Londoño (2007) sostienen que:

El envejecimiento es un proceso natural, universal, que ocurre en todos los seres vivos y se caracteriza por ser irreversible, progresivo, heterogéneo, deletéreo y multifactorial; tiene lugar durante la última etapa del ciclo vital, en donde hay una disminución progresiva de la reserva en la capacidad funcional $y$ en consecuencia una menor habilidad de adaptación a factores del medio ambiente, es una etapa de máxima vulnerabilidad en los aspectos físico, psíquico y social, que puede progresar y convertirse en un anciano frágil, el cual se caracteriza por la pérdida de la funcionalidad, fuerza y reserva fisiológica, con una mayor posibilidad de ser vulnerable a enfermedades y morir. (p. 1073)

Como se puede colegir de la lectura y comprensión del texto anterior, la vejez es la etapa final de la vida, caracterizada por una creciente incapacidad de trabajar y valerse por uno mismo. La historia de la humanidad muestra que, antiguamente, en algunas sociedades, los ancianos eran protegidos por la familia y compartían la alimentación, vivienda y seguridad con el grupo; en otras sociedades, los ancianos no recibían ningún tipo de sustento; hubo también situaciones en que los adultos mayores fueron abandonados y expuestos a la pobreza material y moral. Con la revolución industrial se generó y extendió el trabajo asalariado, surgiendo de este modo la necesidad de nuevas formas alternativas para la atención de los adultos mayores. Esta forma de comprender la humanidad y la etapa de vejez fue el fundamento para la creación de los sistemas de pensiones en las sociedades modernas.

Actualmente, el sistema de pensiones en el Perú cuenta, básicamente, con dos pilares: la primera es de tipo contributivo y de reparto; la segunda también contributiva, pero de capitalización individual. El sistema de reparto se sustenta en el principio de solidaridad intergeneracional, dado que es la generación contribuyente la que financia las pensiones de los adultos mayores que ya no trabajan, 
pero reciben una pensión de jubilación; de manera recíproca y continuada, las pensiones de jubilación de los contribuyentes actuales serán financiados con los aportes o contribuciones de la siguiente generación. En cambio, en el sistema de capitalización, cada trabajador cotiza a una cuenta individual, que se acumula en el tiempo de las aportaciones, de tal modo que las pensiones guardan relación directa con las cotizaciones efectuadas por cada trabajador, durante su vida laboral. En este caso no aparece el componente de solidaridad intergeneracional que caracteriza al sistema de reparto (Olivera, 2009).

En el año de 1993, se implantó en el Perú el Sistema Privado de Pensiones (AFP) con el argumento teórico de que, al constituirse cuentas individuales y capitalizables, un mayor número de trabajadores independientes se incorporarían al sistema de pensiones, ampliando la cobertura del sistema. Sin embargo, transcurrido aproximadamente veintisiete años de funcionamiento de las AFP, la baja cobertura y los bajos niveles de las pensiones de jubilación siguen siendo problemas aún no resueltos (Olivera, 2009).

Hasta antes de la vigencia del Programa Pensión 65, el $23,9 \%$ de los adultos de 65 años a más se encontraba en situación de pobreza; el 7\% de ellos en pobreza extrema y el $16,9 \%$ eran pobres. El $94 \%$ y el $99 \%$ de las personas que vivían en pobreza y pobreza extrema, respectivamente, no contaban con ningún ingreso por pensión de jubilación, hecho que explica el alto porcentaje de adultos mayores que seguían trabajando (HelpAge International, 2016).

Para revertir este panorama, en el año 2011, se crea el Programa Nacional de Asistencia Solidaria, Pensión 65, mediante Decreto Supremo $N^{\circ}$ 081-2011-PCM, cuya finalidad es otorgar protección a los adultos a partir de los 65 años de edad, que carezcan de las condiciones básicas para su subsistencia, entregándoles una subvención económica de 250 soles bimestrales por persona; contribuyendo a cubrir sus necesidades básicas.

A diferencia de los sistemas de las pensiones contributivas, Pensión 65 constituye una primera experiencia de pensiones no contributivas, cuya esencia central es que no requiere contribuciones previas de parte de los potenciales receptores de estas pensiones, porque se sustenta en el enfoque de derechos humanos y ciudadanía. $\mathrm{El}$ argumento teórico en el que se sostiene las pensiones no contributivas afirma que estas pensiones consisten en transferencias monetarias destinadas a fijar un piso o nivel mínimo de ingresos, es decir, generan un incremento en el ingreso per cápita del beneficiario; al incrementarse este ingreso, de acuerdo con la teoría de la función consumo, se espera un incremento proporcional en el consumo y, en consecuencia, debe reducirse los niveles de pobreza entre las personas adultas mayores e, indirectamente, en el resto de la población (Durán, Mendoza y Picado, 2009).

En este contexto, adquiere relevancia económica y social, evaluar el impacto del Programa Pensión 65 sobre un objetivo nacional: la reducción de la pobreza, luego de siete años de haberse implantado e invertido importantes sumas del presupuesto público. En esa línea de acción, el propósito de esta investigación es determinar la relación de las pensiones no contributivas con el nivel de pobreza en los adultos mayores en el Perú, periodo 2012-2018.

$\mathrm{Al}$ respecto, se advierte en varios trabajos de investigación, que existiría correlación entre las pensiones no contributivas y la disminución de la pobreza en los adultos mayores; entre estos trabajos se mencionan los siguientes.

Oliveri (2014) investigó la incidencia de las pensiones no contributivas en el nivel de pobreza en la vejez en América latina. Los resultados obtenidos demuestran que las pensiones no contributivas han reducido de manera significativa los niveles de pobreza extrema en el periodo 2000-2012, así como una incidencia relativamente menor en la reducción de la pobreza moderada. Esto constituye una experiencia eficaz y eficiente de políticas públicas para superar la pobreza y vulnerabilidad de los adultos mayores en América Latina. Entre los países con mejores resultados sobresalen Bolivia, Panamá, Ecuador y Costa Rica; en términos de la reducción de la pobreza extrema, las Pensiones no contributivas explican el $66,1 \%$ de su caída en Panamá, el 56,3\% en Bolivia, el 46,4\% en Ecuador y el $38,7 \%$ en Costa Rica.

Galiani, Gertler y Bando (2014) evalúan los efectos de la pensión no contributiva que otorga el Programa Adultos Mayores de México en el bienestar de los beneficiarios, constituidos por adultos de 70 a más años de edad, del ámbito rural. Este trabajo, según los autores, se desarrolló en un contexto progresivo de creación de planes de pensiones no contributivos en América Latina, como instrumento de lucha contra la pobreza. Los resultados obtenidos muestran que la salud mental de los adultos mayores beneficiarios del programa mejoró significativamente, dado que la depresión se redujo en $12 \%$ en la Escala de Depresión Geriátrica; de similar forma el porcentaje de adultos mayores que realizaban trabajo remunerado disminuyó en 20\%, respecto al nivel de consumo se encontró un aumento promedio del 23\%. Estos resultados nuevamente confirman que las pensiones no contributivas en los países de América Latina sí pueden mejorar el bienestar de los adultos mayores pobres.

Escobar, Martínez y Mendizábal (2013) evaluaron los impactos de la Renta Universal de Vejez - Renta Dignidad 
en el nivel del ingreso per cápita, consumo y pobreza de los hogares bolivianos. Este trabajo fue de tipo no experimental de regresión discontinua, utilizó información de la encuesta nacional urbana y rural del año 2011, que recoge información estadística de los hogares con personas adultas cuyas edades son próximas a los 60 años. Los resultados muestran impactos positivos en los tres indicadores: se determinó un incremento en el ingreso per cápita de Bs 164,58 y que en términos porcentuales representa un incremento de $16,4 \%$ respecto del promedio de ingreso per cápita del grupo de control. Los hogares beneficiarios de la Renta Dignidad incrementaron su consumo per cápita en $15,4 \%$. En cuanto a pobreza monetaria los resultados muestran una reducción de 10,5 puntos porcentuales en el modelo básico y de 13,5 puntos porcentuales en el modelo ampliado; este último con un mayor nivel de explicación y un nivel de confianza del 99\%. Por otro lado, analizando los coeficientes obtenidos según área de residencia, para los tres indicadores, se observa impactos significativos en el área urbana, mientras que, en el área rural si bien se obtiene coeficientes negativos, no son estadísticamente significativos.

Gertler y Galiani, (2016) a solicitud del Ministerio de Economía y Finanzas del Perú, desarrollaron el trabajo titulado Nueva evidencia causal de las pensiones no contributivas en Perú, cuyo objetivo fue conocer los efectos del Programa Nacional de Asistencia Solidaria Pensión 65 en el bienestar de los adultos mayores. Los resultados demostraron impactos positivos en varias dimensiones; entre estos destaca que el $4 \%$ de los adultos mayores dejaron de trabajar, luego de ser admitidos como beneficiarios de Pensión 65; los hogares con al menos un usuario del programa incrementaron su consumo aproximadamente en $40 \%$; finalmente el nivel de depresión (bienestar subjetivo) se redujo en $9 \%$.

Del análisis de las principales conclusiones de los antecedentes presentados, se observa una relación directa entre las pensiones no contributivas y el bienestar de los adultos mayores beneficiarios de estas pensiones en diversos países de América Latina y en Perú en particular.

\section{MATERIALES Y MÉTODOS}

El diseño de este trabajo fue cuantitativo no experimental y de corte longitudinal; de alcance descriptivo, correlacional y explicativo. Descriptivo, en tanto, que se muestra el estado o situación actual de las variables; correlacional porque se establece la relación o asociatividad entre las variables dependientes e independientes; y es explicativo, en la medida de que cada una de las variables independientes explican en algún nivel el comportamiento de la variable dependiente. La población y muestra del estudio estuvo conformada por los adultos mayores en situación de po- breza, beneficiarios del Programa Pensión 65 y que fueron comprendidos en la Encuesta Nacional de Hogares (ENAHO) en el periodo 2012-2018, que en este caso asciende a 158977 personas encuestadas. Los datos utilizados provienen de la base de datos de los módulos Programas Sociales, Sumaria, Educación, Salud, Empleos e Ingresos de la Encuesta Nacional de Hogares de la Instituto Nacional de Estadística e Informática para el periodo 2012-2018.

Las variables consideradas son la pobreza y Pensión 65, como variable explicada y explicativa, respectivamente. Adicionalmente, se han considerado como variables moderadoras: sexo, área geográfica, edad, idioma, trabajo, estado civil, nivel educativo, miembros por hogar y salud.

El modelo econométrico propuesto es un modelo de regresión no lineal.

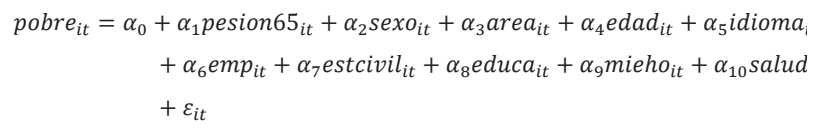

Donde:

pobre $_{i t}=1$ Pobre y 0 No pobre;

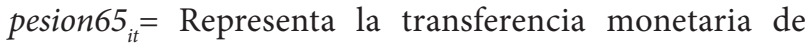
Programa Pensión 65;

sexo $_{i t}=$ Representa el género (hombre y mujer);

area $_{i t}=$ Representa el área geográfica (rural y urbano);

edad $_{i t}=$ Representa la edad en años cumplidos;

idioma $_{i t}=$ Representa el idioma o lengua materna (español u otro);

$c m p_{i t}=$ Representa situación de empleo (ocupado, desocupado o no PEA);

estcivil $l_{i t}=$ Representa estado civil (casado o soltero);

educa $_{\text {it }}=$ Representa nivel educativo (educ. superior, EBR completo o incompleto);

mieho $_{i t}=$ Representa el número de miembros del hogar;

salud $_{i t}=$ Representa estado de salud (padece de alguna enfermedad crónica y no)

$\varepsilon_{i t} \quad=$ Representa el error del modelo.

\section{RESULTADOS}

Los primeros resultados descriptivos nos permiten hacer la caracterización de los adultos mayores en situación de pobreza, a partir de la comparación con los que no lo son, en función a las variables moderadoras consideradas en 
la investigación. El perfil aproximado de un adulto mayor en situación de pobreza evidencia bajo nivel de educación, con una mayor proporción de mujeres, con residencia mayoritariamente en el área rural, de estado civil casado o conviviente, con un idioma nativo aprendido en su niñez, seguido de la población con el español como lengua materna. Laboralmente, estos adultos siguen trabajando a fin de generar ingresos para el hogar y paliar su situación de pobreza, no tienen otra opción que seguir trabajando hasta donde puedan. La figura 1 ilustra de mejor manera el perfil del adulto mayor en situación de pobreza en el Perú.

En la tabla 1, se presentan los coeficientes y efectos marginales de los estimadores asociados al modelo econométrico que predice la probabilidad de que un adulto mayor caiga en situación de pobreza. En este caso se ha utilizado el modelo econométrico no lineal Probit, que se utiliza cuando la variable dependiente es binaria o dummy, es decir que solo puede tomar dos valores.

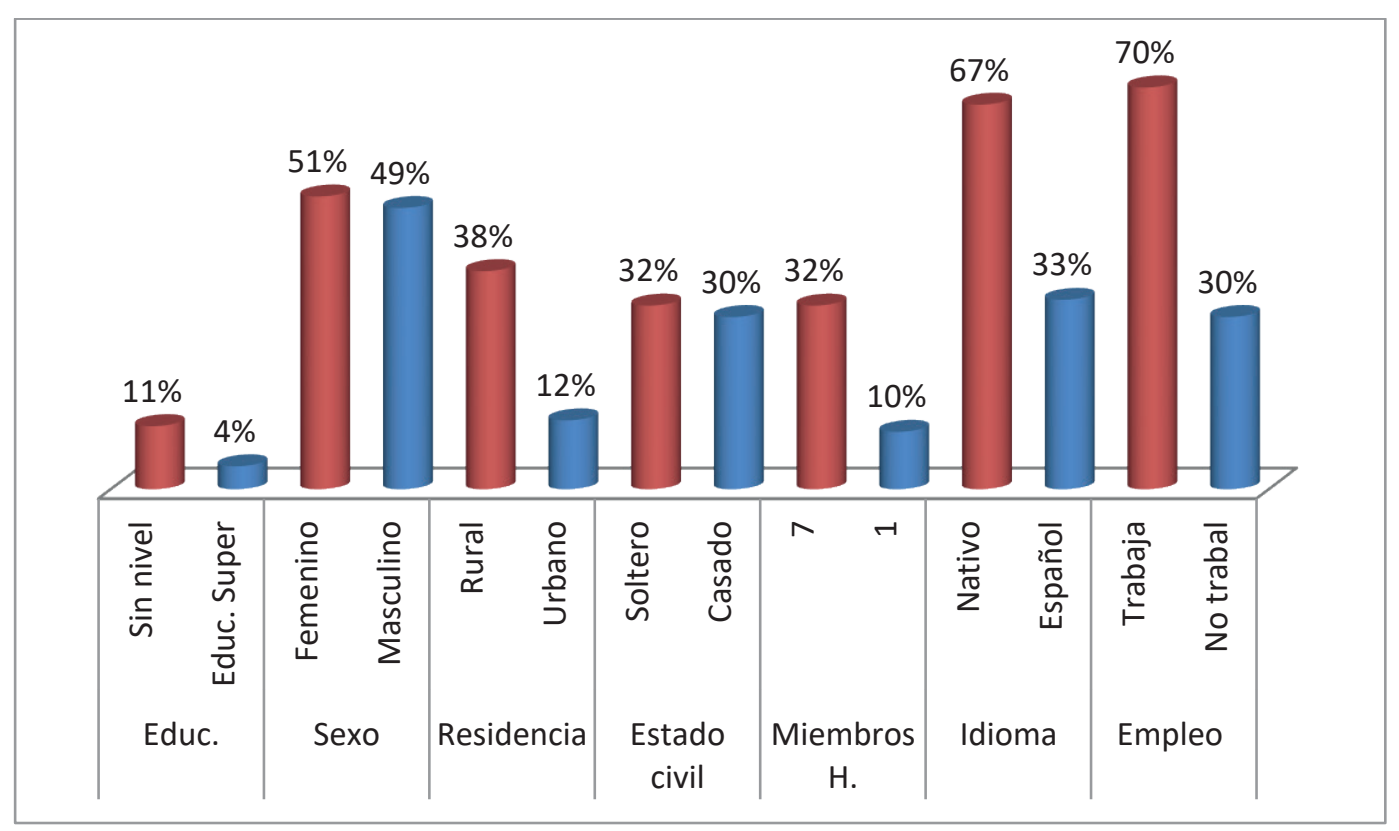

Figura 1. Perfil del adulto mayor en situación de pobreza, Perú 2018.

Fuente: Adaptado de Encuesta Nacional de Hogares 2019, Instituto Nacional de Estadística e Informática, 2019 (https://www.inei.gob.pe/)

Tabla 1

Coeficiente y efectos marginales del modelo PROBIT

\begin{tabular}{ccccccc}
\hline pobre & coef & Efectos marginales & $\mathbf{z}$ & \multicolumn{1}{c}{$\mathbf{P}|\mathbf{z}|$} & \multicolumn{2}{c}{ [95\% Conf. Interval] } \\
\hline pesion65* & $-0,3909805$ & $-0,1059651$ & 16,54 & 0,000 & 0,093411 & 0,118519 \\
sexo* $^{*}$ & 0,0152738 & 0,0034775 & 1,44 & 0,150 & $-0,001252$ & 0,008207 \\
área* & $-0,7074888$ & $-0,1943321$ & $-61,8$ & 0,000 & $-0,200496$ & $-0,188169$ \\
edad & 0,0011641 & 0,0002651 & 3,65 & 0,000 & 0,000123 & 0,000407 \\
idioma* $_{\text {emp* }}^{0,2149047}$ & 0,0524107 & $-16,29$ & 0,000 & $-0,058716$ & $-0,046105$ \\
estcivil* & $-0,0063719$ & $-0,0014530$ & $-0,53$ & 0,594 & $-0,00680$ & 0,003895 \\
educa* & $-0,1115647$ & $-0,0247704$ & $-8,91$ & 0,000 & $-0,030219$ & $-0,019322$ \\
miembh $^{*}$ & $-0,4863205$ & $-0,1107546$ & $-55,4$ & 0,000 & $-0,114673$ & $-0,106836$ \\
salud* & $-0,1543765$ & 0,0351577 & 57,58 & 0,000 & 0,033961 & 0,036354 \\
Constante & $-0,604686$ & $-0,0499624$ & $-19,5$ & 0,000 & $-0,054985$ & $-0,04494$ \\
\hline
\end{tabular}

Fuente: Elaboración propia a partir del paquete estadístico Stata. 
El signo de los coeficientes señala el sentido del efecto de las variables explicativas, es decir que el signo (-) reduce y el signo (+) aumenta el nivel de pobreza. De esta manera, se observa que pensión 65, área de residencia, empleo, estado civil, educación y salud contribuyen a la reducción del nivel de la pobreza en los adultos mayores.

El efecto marginal expresa el cambio en la variable dependiente provocado por un cambio unitario en la variable independiente, manteniendo el resto constante. Ser beneficiario del programa Pensión 65 aumenta en 0,10597 la probabilidad de no ser pobre, en relación con aquel que no es beneficiario de este programa; ser de sexo femenino incrementa en 0,00348 la probabilidad de ser pobre, en relación al de sexo masculino; vivir en el área urbana reduce en 0,19433 la probabilidad de ser pobre respecto al que vive en el área rural; el aumento unitario de la edad aumenta en 0,00027 la probabilidad de ser pobre, manteniendo constantes las otras variables; tener un idioma nativo como primera lengua aumenta 0,05241 la probabilidad de ser pobre respecto a otros idiomas como primera lengua; estar en condición de ocupado en un empleo disminuye en 0,001453 la probabilidad de ser pobre respecto a estar desocupado; estar casado reduce en 0,02477 la probabilidad de ser pobre respecto a estar soltero; un año adicional de estudios reduce en 0,11075 la probabilidad de ser pobre respecto al nivel anterior; el incremento en una unidad de los miembros del hogar aumenta en 0,03516 la probabilidad de ser pobre, manteniendo constantes las otras variables.

Con los efectos marginales estimados, se propone el modelo econométrico que explique y estime la probabilidad de pobreza de un adulto mayor, entre los años 2012-2018.

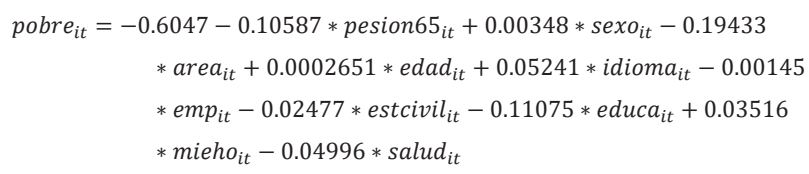

A la luz de los resultados obtenidos, se sostiene que el Programa Solidario Pensión 65, de modo general, tuvo un efecto positivo en la reducción de la pobreza en los adultos mayores en el Perú, durante el periodo 2012-2018. Se encontró evidencia estadística de una asociación inversa y significativa, entre ambas variables: los adultos mayores beneficiarios del Programa Pensión 65, tienen un 10,5\% más de probabilidad de superar la situación de pobreza, en comparación con los adultos mayores que no reciben dicha pensión.

En base al análisis del modelo econométrico estimado, se han identificado variables moderadoras que contribuyen a reducir la pobreza, como el área de residencia, empleo, estado civil, educación, y salud. Vivir en el área urbana reduce la probabilidad de ser pobre en $19,43 \%$ frente a los que viven en el área rural; resultado que tiene sentido lógico, en tanto que el área urbana ofrece mejores condiciones de vida, en comparación al área rural.

El empleo es otra variable que reduce la probabilidad de ser pobre en la vejez, situación que se puede comprender también como la necesidad de seguir trabajando durante la vejez a falta de ahorros o pensión de jubilación. Respecto al estado civil, se evidencia que los casados o convivientes tienen mayores posibilidades de superar la pobreza, lo cual es coherente con la teoría, en tanto que la institución familiar tiene como fundamento proveer protección, seguridad y soporte emocional a sus miembros; mientras que los adultos mayores que no constituyeron familias, posiblemente, vivan su vejez aislados y solitarios, sin el apoyo material y emocional de la familia, expuestos en mayor medida a la pobreza. El nivel de educación es otra variable que influye en la probabilidad de ser pobre en la vejez, un año adicional de educación reduce el riesgo de ser pobre en $11 \%$; este resultado también es coherente con la teoría del capital humano o con el enfoque de capacidades.

Finalmente, el buen estado de salud, según el modelo, reduce la probabilidad de ser pobre en $4,9 \%$; resultado consistente con la teoría, en tanto que una persona o población saludable goza de bienestar fisiológico y emocional.

\section{DISCUSIÓN}

Oliveri (2014), en su tesis de maestría Adultos Mayores en América Latina: Pensiones No Contributivas y Pobreza, cuyos resultados confirman que las pensiones no contributivas inciden positivamente en la reducción de la pobreza en la vejez, menciona como casos destacables que las pensiones no contributivas explican la reducción de la pobreza en Panamá en un 66,1\%; en Bolivia en un 56,3\%; en Ecuador en un 46,4\% y en Costa Rica en 38,7\%. Por su parte Escobar, Martínez y Mendizábal (2013) al evaluar los impactos de la Renta Universal de Vejez - Renta Dignidad de Bolivia encuentran que la pobreza se reduce en 10,5 puntos porcentuales, en el modelo básico; y de 13,5 puntos porcentuales, en el modelo ampliado. Por otro lado, los coeficientes obtenidos según área de residencia permiten observar impactos significativos en el área urbana, mientras que, en el área rural, si bien se obtiene coeficientes negativos, los mismos no son estadísticamente significativos. Estos resultados guardan relación con los obtenidos en esta investigación, en el sentido de que los beneficiarios del Programa Pensión 65 tienen 10,5\% más de probabilidad de superar la pobreza, frente a los adultos no beneficiarios del programa; y que la pobreza afecta más a los que viven en el área rural del Perú. En el plano nacional, Gertler y Galiani (2016) evalúan los impactos del 
Programa Pensión 65 en el bienestar de los adultos mayores; los resultados de este trabajo demuestran impactos positivos en varias dimensiones a favor de los adultos mayores que reciben la transferencia monetaria del programa Pensión 65. Entre estos impactos se menciona que 4\% de los adultos mayores dejaron de trabajar, luego de ser admitidos como beneficiarios de Pensión 65; los hogares con al menos un usuario del programa incrementaron su consumo aproximadamente en $40 \%$; finalmente el nivel de depresión (bienestar subjetivo) se redujo en 9\%. En general, los resultados de la citada investigación están en la misma línea de los resultados obtenidos en este trabajo. En conclusión, los resultados de esta investigación confirman que el Programa Pensión 65 constituye una política pública eficaz y eficiente para disminuir el nivel de pobreza y vulnerabilidad en la vejez en el Perú; asimismo, abonan a favor de una política de seguridad social que incorpore las pensiones no contributivas como un tercer pilar del sistema de pensiones, fundamentado en los derechos humanos y ciudadanía.

\section{REFERENCIAS}

Bando, R., Gertler, P., y Galiani, S. (2014). Noncontributory pensions. Inter-American Development Bank. Working Paper Series; 517. Recuperado de https://publications.iadb.org/publications/english/document/ Non-contributory-pensions.pdf.

Castro, S. (2013). Pobreza, minería y conflictos socioambientales en el Perú. Lima, Perú. INTEPUCP.

Durán, F., Mendoza, W., y Picado, G. (2009). Proyecciones demográficas y financieras para el análisis de la viabilidad de pensiones no contributivas en el Perú. En Clark, F., Durán, F., Mendoza De Souza, W., Picado, G., Morón, E., Rostagno, M., Arnillas, F. y Sánchez, C. (Eds.). Envejecimiento con dignidad: Pensiones no contributivas para reducir la pobreza en el Perú (pp. 37-87). Recuperado de http://www.helpagela.org/silo/files/envejecimiento-con-dignidad-per.pdf

Escobar, F., Martínez, S., y Mendizábal, W. (2013). El Impacto de la Renta Dignidad: Política de Redistribución del Ingreso, Consumo y Reducción de la Pobreza en Hogares con Personas Adultas Mayores. Recuperado de http://www.udape.gob.bo/evaluaciondeimpacto/12_ Documento_Impacto\%20Renta\%20Dignidad.pdf
Galiani, S., Gertler, P. y Bando, R. (2014). Non-contributory pensions. Inter-American Development Bank. Working Paper (517), 1-27. Recuperado de https:// publications.iadb.org/publications/english/document/ Non-contributory-pensions.pdf

Gertler, P. y Galiani, S. (2016). Evaluación de Impacto del Programa Pensión 65: Nueva evidencia causal de las pensiones no contributivas en Perú. Recuperado de https://www.mef.gob.pe/contenidos/presu_publ/ppr/ eval_indep/informe_resultados_pension65.pdf

HelpAge International. (2016). Protección social a personas adultas mayores en pobreza extrema en Perú Pensión 65 y el sistema de pensiones no contributivas. Recuperado de https:/www.helpage.org/silo/files/ social-protection-for-older-people-in-extreme-poverty-in-peru-spanish.pdf

Instituto Nacional de Estadística e Informática. (2019). Encuesta Nacional de Hogares 2019. Recuperado de: https://www.inei.gob.pe/

Modigliani, F. (1986). Life cycle, Individual Thrift, and the Wealth of Nations. American Economic Review, 76(3), 297-313.

Ocampo, J. y Londoño, I. (2007). Ciclo Vital Individual: Vejez. Revista de la Asociación Colombiana de Gerontología y Geriatría, 21(3), 1072- 1084. Recuperado de http:// acgg.org.co/pdf/pdf_revista_07/21-3.pdf\#page=36

Olivera, J. (2009). Recuperando la Solidaridad en el Sistema de Pensiones Peruano: Una propuesta de reforma. Recuperado de http://cies.org.pe/sites/default/files/ investigaciones/recuperando-la-solidarida-en-el-sistema-de-pensiones-peruano.pdf

Oliveri, M. (2014). Adultos Mayores en América Latina: Pensiones No Contributivas y Pobreza (Tesis de maestría, Universidad Nacional de La Plata, Argentina). Recuperada de http://www.depeco.econo.unlp.edu.ar/wp/ wp-content/uploads/2017/08/115-tesis-Oliveri.pdf 